\title{
The mass function of dense molecular cores and the origin of the IMF ${ }^{\star}$
}

\author{
J. Alves ${ }^{1}$, M. Lombardi ${ }^{2, \star \star}$, and C. J. Lada ${ }^{3}$ \\ 1 Calar Alto Observatory - Centro Astronómico Hispano Alemán, C/Jesús Durbán Remón 2-2, 04004 Almeria, Spain \\ e-mail: jalves@caha.es \\ 2 European Southern Observatory, Karl-Schwarzschild-Str. 2, 85748 Garching, Germany \\ e-mail: mlombard@eso.org \\ 3 Harvard-Smithsonian Center for Astrophysics, Mail Stop 72, 60 Garden Street, Cambridge, MA 02138, USA \\ e-mail: clada@cfa.harvard.edu
}

Received 12 August 2006 / Accepted 5 December 2006

\begin{abstract}
Context. Stars form in the cold dense cores of interstellar molecular clouds and the detailed knowledge of the spectrum of masses of such cores is clearly a key for the understanding of the origin of the IMF. To date, observations have presented somewhat contradictory evidence relating to this issue.

Aims. In this paper we propose to derive the mass function of a complete sample of dense molecular cores in a single cloud employing a robust method that uses uses extinction of background starlight to measure core masses and enables the reliable extension of such measurements to lower masses than previously possible.

Methods. We use a map of near-infrared extinction in the nearby Pipe dark cloud to identify the population of dense cores in the cloud and measure their masses.

Results. We identify 159 dense cores and construct the mass function for this population. We present the first robust evidence for a departure from a single power-law form in the mass function of a population of cores and find that this mass function is surprisingly similar in shape to the stellar IMF but scaled to a higher mass by a factor of about 3 . This suggests that the distribution of stellar birth masses (IMF) is the direct product of the dense core mass function and a uniform star formation efficiency of $30 \% \pm 10 \%$, and that the stellar IMF may already be fixed during or before the earliest stages of core evolution. These results are consistent with previous dust continuum studies which suggested that the IMF directly originates from the core mass function. The typical density of $\sim 10^{4} \mathrm{~cm}^{-3}$ measured for the dense cores in this cloud suggests that the mass scale that characterizes the dense core mass function may be the result of a simple process of thermal (Jeans) fragmentation.
\end{abstract}

Key words. ISM: structure - dust, extinction - stars: formation - stars: luminosity function, mass function ISM: evolution - ISM: clouds

\section{Introduction}

The stellar initial mass function (IMF) is one of the most fundamental distributions in astrophysics. Detailed knowledge of its functional form and how this form varies in space and time is necessary to predict and understand the evolution of stellar systems, from clusters to galaxies. Nevertheless, the origin of the stellar IMF remains one of the major unsolved problems of modern astrophysics.

Stars form in interstellar molecular clouds and the detailed knowledge of the spectrum of structure in such clouds is likely a key for the understanding of the origin of the IMF. What is the relation between cloud structure and the IMF? To date, observations have presented somewhat contradictory evidence relating to this issue. Early $\mathrm{CO}$ observations suggested that the mass spectrum of molecular cloud structure is well described by

* Table of core positions is only available in electronic form at the CDS via anonymous ftp to cdsarc.u-strasbg.fr (130.79.128.5) or via

http://cdsweb.u-strasbg.fr/cgi-bin/qcat?J/A+A/462/L17

$\star \star$ On leave from University of Milan, Department of Physics, via Celoria 16, 20133 Milan, Italy. a single power-law (Blitz 1993; Kramer et al. 1998; Heithausen et al. 1998), specifically, $\mathrm{d} N(m) / \mathrm{dlog} m \sim m^{-0.7}$ for structures (clumps) with a wide range of masses that apparently extends from a fraction a solar mass to $1000 M_{\odot}$. This functional form differs from the form of the stellar IMF in two fundamental ways.

First, the stellar IMF cannot be described by a single powerlaw (scale-free) function. The general consensus of observational studies is that the stellar IMF is best described by a series of power-law segments that overall is qualitatively similar in shape to a log-normal function (Miller \& Scalo 1979; Kroupa 2002; Muench et al. 2001). In particular, the IMF is characterized by a broad peak which spans roughly the interval of $0.1-0.6 M_{\odot}$ and falls off rapidly on each side of the peak. Between 0.6 and $10 M_{\odot}$ the IMF decreases in a power-law fashion similar to that originally derived by Salpeter (1955). The fact that the IMF displays a peak and is not scale-free is of great significance for star formation since it indicates that there is a characteristic mass resulting from the star formation process of around 0.2-0.3 $M_{\odot}$ (e.g., Larson 2005).

Second, above about a solar mass the slope of the stellar IMF is steeper (i.e., $\mathrm{d} N(m) / \mathrm{d} \log m \sim m^{-1.35}$ ) than the cloud 


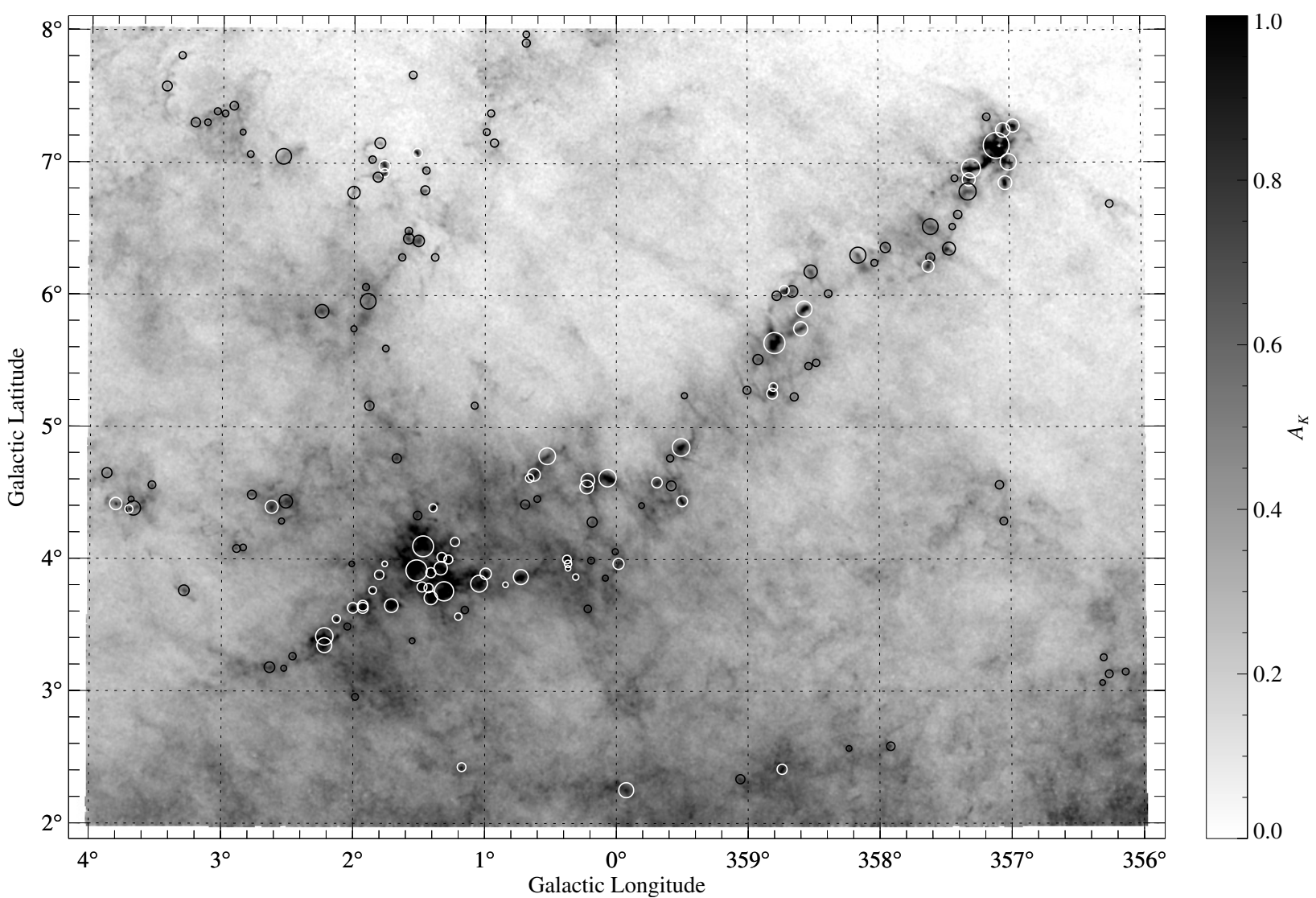

Fig. 1. Dust extinction map of the Pipe nebula molecular complex from Lombardi et al. (2006). This map was constructed from near-infrared observations of about 4 million stars in the background of the complex. Approximately 160 individual cores are identified within the cloud and are marked by an open circle proportional to the core radius. Most of these cores appear as distinct, well separated entities.

mass spectrum. This latter difference implies a significant physical difference in the two distributions. For the stellar IMF, the bulk of the (stellar) mass is tied up in low mass objects while for clouds the bulk of the mass is tied up in the most massive objects. A consequence of this difference is that in order to produce the stellar IMF from the cloud mass spectrum, a transformation must take place during the process of star formation. It has been suggested that outflows generated during the protostellar stages of star formation provide a natural feedback to collapse/infall limiting the final mass of a protostellar object (Shu et al. 1987) and perhaps providing the mechanism for transforming the form of the cloud mass spectrum into the form of the stellar mass spectrum (Lada \& Lada 2003). However, it is not clear how such a process could produce a peak, or a characteristic scale for stellar masses. Moreover, the comparison of the $\mathrm{CO}$ core mass function and the IMF may not be relevant since $\mathrm{CO}$ does not trace the dense component of the molecular gas within which stars actually form (Lada 1992).

Indeed, a different picture appears to emerge when observations of dense gas are considered. Typically only about $10 \%$ or less of the mass of a star-forming molecular cloud is in the form of dense (i.e., $n(\mathrm{H} 2) \sim 10^{4} \mathrm{~cm}^{-3}$ ) gas and this gas appears to be organized into discrete cores within which stars form. Tachihara et al. (2002) and Onishi et al. (2002), using $\mathrm{C}^{18} \mathrm{O}$ and $\mathrm{H}^{13} \mathrm{CO}^{+}$ as tracers of dense gas, suggest that the stellar and core mass distributions are similar. Recent observations of dust continuum emission originating from such dense cores has enabled the construction of the dense core mass function (DCMF) in a number of nearby molecular cloud complexes. For cores with masses in excess of $\sim 0.5 M_{\odot}$ the derived mass spectra appear to be described by a single power-law, similar to the CO cores but with a relatively steep slope $(-1.1$ to -1.6 , in log mass units) similar to the that of the stellar IMF (Motte et al. 1998; Testi \& Sargent 1998; Johnstone et al. 2000, 2001; Motte et al. 2001; Beuther $\&$ Schilke 2004; Stanke et al. 2006). Moreover, in one example, the $\rho$ Ophiuchi cloud, the core mass spectrum exhibited possible evidence of a flattening or break near a mass of about $0.5 M_{\odot}$, also similar to the stellar IMF (Motte et al. 1998). In another cloud, NGC 1333, measurements of dust emission produced a core mass spectrum between 0.1 and $0.5 M_{\odot}$ with a slope of approximately -0.4 (Sandell \& Knee 2001), similar to that universally derived for less-dense gas traced by $\mathrm{CO}$ emission in other clouds but also consistent with the apparent break and assumed flattening below $0.5 M_{\odot}$ of the DCMF of the Ophiuchi dark cloud mentioned above. The observed similarity between the slopes of the DCMF derived from millimeter-wave dust emission studies and the slope of the IMF above $0.5 M_{\odot}$ has been taken as evidence that the individual dense cores are the direct precursors of new stars and moreover that the stellar IMF is completely specified by the fragmentation process in molecular clouds. In addition, the possible flattening of the DCMF near $0.5 M_{\odot}$ implies a high star formation efficiency (SFE) for dense gas (about $100 \%$ ). In this case there is no need for a mass transformation from the DCMF to the IMF. The characteristic scale of stellar mass demanded by the stellar IMF is set by the fundamental physics of cloud fragmentation (Shu et al. 2004; Larson 2005). Which picture is correct?

In order to bring new insight to this issue we used an independent method of identifying and measuring the masses of dense cores. This method uses precise infrared measurements of dust extinction toward stars background to a molecular cloud (the NicE(R) method, Lada et al. 1994; Alves et al. 1998, 2001; 
Lombardi \& Alves 2001). Such measurements are free from many of the complications and systematic uncertainties that plague molecular-line or dust emission data and thus enable robust maps of cloud structure to be constructed. We used data from the recent wide-field extinction map of the Pipe Nebula constructed by Lombardi et al. (2006), hereafter LAL06, using 2MASS data. The Pipe nebula is a virtually unstudied nearby molecular cloud complex (Onishi et al. 1999; LAL06), at a distance of about $130 \mathrm{pc}$ and with a total mass of $\sim 10^{4} M_{\odot}$.

\section{Observations}

The details of the extinction study are described in LAL06. Briefly, this molecular complex was selected because 1) this is one of the closest to Earth complexes of this size and mass, 2) it is particularly well positioned along a relatively clean line of sight to the rich star field of the Galactic bulge, which given the close distance of the Pipe nebula allowed us to achieve spatial resolutions of $\sim 0.03 \mathrm{pc}$, or about 3 times smaller the typical dense core size, and 3) it exhibits very low levels of star formation suggesting that its dense cores likely represent a fair sample of the initial conditions of star formation. LAL06 applied a 3-band (1.25 $\mu \mathrm{m}, 1.65 \mu \mathrm{m}, 2.2 \mu \mathrm{m})$ optimized version of this extinction method, the NICER method (Lombardi \& Alves 2001), to about 4 million stars background to the Pipe nebula complex to construct a $6^{\circ} \times 8^{\circ}$ dust column density map of this complex, presented in Fig. 1. Because of the high dynamic range in column density achieved by this map $\left(3 \sigma \sim 0.5<A_{\mathrm{V}}<24\right.$ mag or $10^{21}<N_{\mathrm{H}}<5 \times 10^{22} \mathrm{~cm}^{-2}$ ), cores are easily visually identified as high contrast peaks embedded on rather smooth but variable background (see Fig. 1). Unfortunately, because of the high dynamic range and variable background, traditional source extraction algorithms based on thresholding fail to identify these objects in a coherent way. An alternative approach is to extract cores based on their sizes, using a multi-scale algorithm. For this study we used a algorithm developed by Vandame (2006, private comm.), which, in brief, uses the wavelet transform of the image to first identify and then reconstruct the dense cores ${ }^{1}$. This step defines the projected core boundaries.

Masses are derived by integrating the extinction map over the area of each core and multiplying by the standard gas-to-dust ratio. The final Pipe core sample has 159 objects with effective diameters between 0.1 and $0.4 \mathrm{pc}$ (median size is $0.18 \mathrm{pc}$ ) and peak extinctions that range from 3.0 to 24.3 visual magnitudes (mean extinction is $8.4 \mathrm{mag}$ ). The derived core masses range between 0.5 to $28 M_{\odot}$. The assessment of sample completeness is non trivial because of the variable background. Nevertheless, the completeness should not be dominated by confusion but sensitivity, as the mean separation between cores, even in the clustered regions, is well above the resolution of the map. We

\footnotetext{
${ }^{1}$ Object identification in wavelet space: for a given scale $i$, structures are isolated with classical thresholding at $3 \sigma_{i}$ with $\sigma_{i}$ being the noise amplitude at scale $i$. A structure at scale $i$ is connected with a structure at scale $i+1$ if its local maxima drops in the structure at scale $i+1$. The size scales considered were $2^{\prime}, 4^{\prime}$, and $8^{\prime}(0.08 \mathrm{pc}, 0.15 \mathrm{pc}$, and $0.30 \mathrm{pc})$. One then builds a 3D distribution of significant structures $(x$, $y$, and $i$ ). The algorithm developed by Vandame (2006, private comm.) offers rules that split the $3 \mathrm{D}$ distribution into independent trees corresponding to one core and its corresponding hierarchical details. Object reconstruction: This same algorithm performs a complex iterative reconstruction of the cores following the trees defined in the previous step. The final "cores only" image is validated by subtracting it from the extinction map, effectively creating a smooth image of the variable background.
}

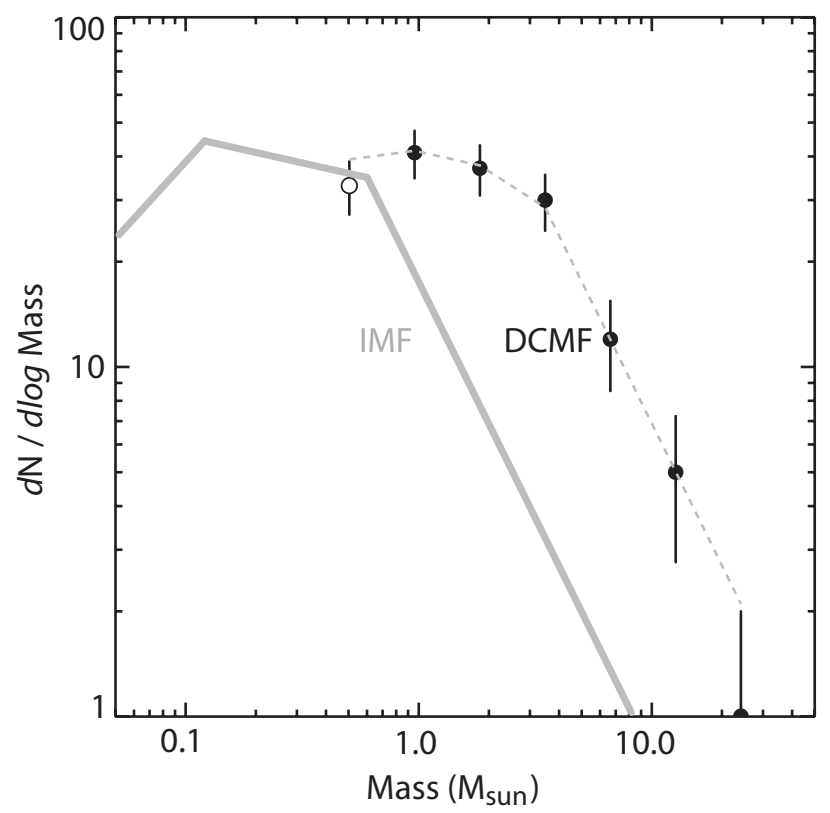

Fig. 2. Mass function of dense molecular cores plotted as filled circles with error bars. The grey line is the stellar IMF for the Trapezium cluster (Muench et al. 2002). The dashed grey line represents the stellar IMF in binned form matching the resolution of the data and shifted to higher masses by about a factor of 4 . The dense core mass function is similar in shape to the stellar IMF function, apart from a uniform star formation efficiency factor.

estimate, conservatively, that the sample is $90 \%$ complete at about $1 M_{\odot}$. The mean diameter of a $1 M_{\odot}$ core is $\sim 0.2 \mathrm{pc}$, i.e., about six times the resolution of the map.

\section{Results: The Dense Core Mass Function (DCMF)}

The dense core mass function we derive from the above observations is presented in Fig. $2^{2}$. For comparison we plot the Trapezium cluster stellar IMF as a grey solid line (Muench et al. 2002). This IMF consists of 3 power law segments with breaks and $0.6 M_{\odot}$ and $0.12 M_{\odot}$. We find that the DCMF for the Pipe Nebula is surprisingly similar in shape to the stellar IMF. Specifically, both distributions are characterized by a Salpeterlike power-law (Salpeter 1955) that rises with decreasing mass until reaching a distinct break point, this is then followed by a broad peak. Although similar in shape, the stellar and core mass functions are characterized by decidedly different mass scales. The grey dashed line in Fig. 2 is not a fit to the data but simply the stellar IMF in binned form matched to the resolution of the data, and shifted by a factor of 4 to the higher masses. The break from the Salpeter-like slope seems to occur between 2 and $3 M_{\odot}$ for the cloud cores instead of the $0.6 M_{\odot}$ for the stellar case (Muench et al. 2002).

The DCMF in Fig. 2 likely suffers from two sources of uncertainty: 1) the individual core masses are likely upper limits to the true values since we made no corrections for the local extended background on which most cores are embedded, and 2) the particular binning we chose may not produce the most accurate representation of the underlying mass distribution. To address point 1) we estimated a lower limit to the true core masses (and the DCMF) by subtracting from each core its local background. We then constructed a background subtracted DCMF and found

\footnotetext{
2 The full dataset is available in electronic form at the CDS.
} 


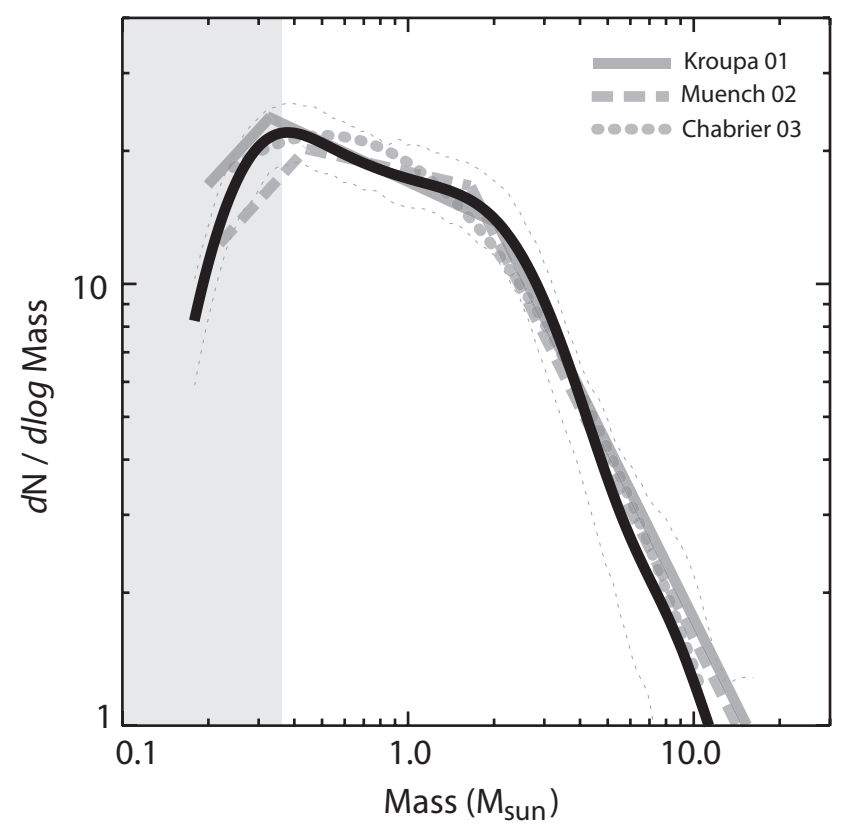

Fig. 3. Probability density function of core masses (black) for the background subtracted sample. The grey region indicates sample incompleteness while the two thin dashed line indicates the $90 \%$ confidence limits. The core mass distribution seems to be characterized by two power-laws and a well defined break point at around $2 M_{\odot}$. Also plotted are the field star IMFs of Kroupa (2001) (solid grey) and Chabrier (2003) (dotted grey), and the Muench et al. (2002) (dashed grey) IMF all scaled up by a factor of $\sim 3$ in mass.

that the form of the mass distribution was maintained although the new distribution was shifted towards the low masses. To address point 2) we compute a better functional representation for the DCMF using a probability density estimator that does not require data binning. We used a gaussian kernel estimator with a window width of 0.15 in units of log mass (Silverman 1986).

We present in Fig. 3 the probability density function for the core masses (background subtracted). As already suggested in Fig. 2, the core mass distribution seems to be characterized by two power-laws and a well defined break point at $\sim 2 M_{\odot}$. For comparison we also present the field star IMF determined by Kroupa (2001) (solid grey) and Chabrier (2003) IMF (dotted grey), and the IMF for the young Trapezium cluster (dashed grey) (Muench et al. 2002) already present in Fig. 2. All these stellar IMFs were shifted to higher masses by an average scale factor of about 3 . The point of this comparison is to simply illustrate the overall similarity between different stellar IMFs from different environments and constructed in different ways, and the DCMF of the Pipe Nebula. Considering the overall uncertainties, apparent differences between the different distributions are likely not significant. While Fig. 3 presents the background subtracted core sample, the non background subtracted sample is virtually identical in shape, although shifted towards higher masses.

\section{Discussion and conclusions}

The close similarity in shape between the DCMF and the IMF supports a general concept of a 1-to-1 correspondence between the individual dense cores and soon to be formed stars. In this respect our observations are consistent with and appear to confirm the results of dust continuum surveys in other clouds. However, our study provides the first robust evidence for a departure from a single power law in the core mass function. We find that the location of the break indicates that there is a factor of about 3 difference in mass scale between the two distributions, which implies that a uniform SFE, that we estimate to be $30 \% \pm 10 \%$, will likely characterize the star formation in these dense cores, across the entire span of (stellar) mass. This efficiency is very similar to the maximum estimated for young embedded clusters (Lada \& Lada 2003).

It is well known that the generation of a powerful outflow is a natural consequence of the formation of a protostar in a dense core (Lada 1985). It has been long suspected that such outflows could be very disruptive to surrounding material and could play an important role in limiting the mass which can accrete onto a protostar (Shu et al. 1987, 1991). Matzner \& McKee (2000) have theoretically investigated the disruption of dense cores by protostellar outflows and have concluded that outflow disruption of individual cores can produce SFEs in the range of $25-75 \%$. Moreover they found that SFE is independent of stellar mass, therefore the stellar and core mass functions should be of similar overall functional form. Recently, Shu et al. (2004) have developed a self-consistent analytical theory for the origin of the stellar IMF incorporating the feedback from outflows. In their picture, cores are formed from magnetized turbulent gas and are disrupted by outflows with a resulting SFE predicted to be $\approx 30 \%$. The close correspondence of this prediction with our estimated SFE for the Pipe cores may implicate outflows as the key mechanism for core disruption and for setting the final masses of the protostellar objects. Shu et al. (2004) also predicted both a slope for the power-law portion of the core mass function and a location of the break from the power-law form that are very similar to those we measure in the Pipe Nebula.

A number of other theoretical calculations have also produced DCMFs similar in shape to that found here (Padoan \& Nordlund 2002; Bonnell et al. 2006; Elmegreen 2006). In particular, numerical simulations of turbulent clouds predict DCMF shapes similar to the stellar IMF. However it is difficult to compare these calculations in more detail to the derived Pipe DCMF because, unlike the Shu et al. (2004) model, the mass scales for these DCMFs are presently unconstrained and appear to be dependent on model parameters, such as the numerical resolution of the simulations as well as the Mach number of turbulence which are difficult to quantify observationally (e.g., Klessen 2001; Ballesteros-Paredes \& Mac Low 2002; Gammie et al. 2003). Nonetheless we expect that with appropriate modification of model parameters such models could also be made consistent with the observations.

These considerations suggest that apart from a uniform efficiency factor, which is likely fixed by the generation of an outflow, the birth mass of a star could to be completely predetermined by the mass of the dense core in which it is born.

The existence of a characteristic mass in the stellar IMF does suggests a characteristic mass scale is produced by the physical process of star formation. The physical origin of this mass scale has not been clear although it has been long known that thermal (Jeans) fragmentation can produce a mass scale for star formation (Larson 1985, 1986). For a pressure bounded core the appropriate mass scale is the critical Bonnor-Ebert mass,

$M_{\mathrm{BE}} \approx 1.15 \times\left(n / 10^{4}\right)^{-1 / 2} \times(T / 10)^{3 / 2}$

where $T$ is the gas temperature and $n$ its volume density. The characteristic mass scale suggested by the stellar IMF is about $0.3 M_{\odot}$. For a typical dark cloud of temperature of $10 \mathrm{~K}$, the corresponding density scale would need to be about $1.5 \times 10^{5} \mathrm{~cm}^{-3}$. 
This is significantly higher than the measured average mean density of the Pipe cores of $8 \times 10^{3} \mathrm{~cm}^{-3}$. However, if the characteristic stellar mass scale is set by the characteristic mass of the DCMF, which is a factor of $\sim 3.3$ higher than that of the stellar IMF, then the corresponding critical density is $\sim 1.3 \times 10^{4} \mathrm{~cm}^{-3}$ much closer to that observed for the Pipe dense cores. This correspondence suggests that the fragmentation scale for the DCMF and ultimately the stellar IMF may be set by a process of thermal (Jeans-like) fragmentation, perhaps modified somewhat by ambipolar diffusion and/or turbulence. Interestingly, from the recent analysis of Spitzer observations of the spatial distribution of protostellar objects in NGC 2264, Teixeira et al. (2006) found evidence for Jeans like fragmentation to be occurring in that star forming region.

We note that the mean densities of the cores in our study range between $5 \times 10^{3}$ and $2 \times 10^{4} \mathrm{~cm}^{-3}$ (for the background subtracted case). This range is lower than that $\left(3 \times 10^{4-5} \mathrm{~cm}^{-3}\right)$ usually associated with typical star forming cores and even the starless cores typically traced by dust continuum observations (Motte et al. 1998; Johnstone et al. 2001; Kirk et al. 2005). Because extinction observations are more sensitive to lower column densities we are able to probe lower mass and density regimes than those probed by current dust continuum studies. If the Pipe Nebula cores are to form stars, the relatively low mean densities they currently possess may indicate that they are in a very early stage of development. This notion is somewhat strengthened by the absence of significant star formation activity in the Pipe nebula complex. Apparently, the functional form of the stellar IMF may be fixed during or before the earliest stages of core evolution.

The results of this letter suggest that the distribution of stellar birth masses over essentially the entire range of stellar mass is the product of the dense core mass function and a uniform SFE of $30 \% \pm 10 \%$. Hence, the question of the origin of the IMF is the question of the origin of the DCMF, itself a result of the physical process of cloud fragmentation/coalescence. The mass scale characterizing the DCMF may result from a simple process of thermal (Jeans-like) fragmentation in the molecular gas.

Acknowledgements. C.J.L. acknowledges support from NASA ORIGINS Grant NAG 5-13041. This publication makes use of data products from the Two Micron All Sky Survey, which is a joint project of the University of Massachusetts and the Infrared Processing and Analysis Center/California Institute of Technology, funded by the National Aeronautics and Space Administration and the National Science Foundation.

\section{References}

Alves, J., Lada, C. J., Lada, E. A., Kenyon, S. J., \& Phelps, R. 1998, ApJ, 506, 292

Alves, J., Lada, C. J., \& Lada, E. A. 2001, Nature, 409, 159

Ballesteros-Paredes, J., \& Mac Low, M.-M. 2002, ApJ, 570, 734

Beuther, H., \& Schilke, P. 2004, Science, 303, 1167

Blitz, L. 1993, in Protostars and Planets III, 125-161

Bonnell, I. A., Larson, R. B., \& Zinnecker, H. 2006, in Protostars and Planets V Chabrier, G. 2003, ApJ, 586, L133

Elmegreen, B. G. 2006, in ASP Conf. Ser. 7th Pacific Rim Conference on Stellar Astrophysics

Gammie, C. F., Lin, Y.-T., Stone, J. M., \& Ostriker, E. C. 2003, ApJ, 592, 203

Heithausen, A., Bensch, F., Stutzki, J., Falgarone, E., \& Panis, J. F. 1998, A\&A, 331, L65

Johnstone, D., Fich, M., Mitchell, G. F., \& Moriarty-Schieven, G. 2001, ApJ, 559,307

Johnstone, D., Wilson, C. D., Moriarty-Schieven, G., et al. 2000, ApJ, 545, 327

Kirk, J. M., Ward-Thompson, D., \& André, P. 2005, MNRAS, 360, 1506

Klessen, R. S. 2001, ApJ, 556, 837

Kramer, C., Alves, J., Lada, C., et al. 1998, A\&A, 329, L33

Kroupa, P. 2001, MNRAS, 322, 231

Kroupa, P. 2002, Science, 295, 82

Lada, C. J. 1985, ARA\&A, 23, 267

Lada, C. J., \& Lada, E. A. 2003, ARA\&A, 41, 57

Lada, C. J., Lada, E. A., Clemens, D. P., \& Bally, J. 1994, ApJ, 429, 694

Lada, E. A. 1992, ApJ, 393, L25

Larson, R. B. 1985, MNRAS, 214, 379

Larson, R. B. 1986, in Stellar Populations, ed. C. A. Norman, A. Renzini, \& M. Tosi, 101

Larson, R. B. 2005, MNRAS, 359, 211

Lombardi, M., \& Alves, J. 2001, A\&A, 377, 1023

Lombardi, M., Alves, J., \& Lada, C. J. 2006, A\&A, 454, 781 (LAL06)

Matzner, C. D., \& McKee, C. F. 2000, ApJ, 545, 364

Miller, G. E., \& Scalo, J. M. 1979, ApJS, 41, 513

Motte, F., Andre, P., \& Neri, R. 1998, A\&A, 336, 150

Motte, F., André, P., Ward-Thompson, D., \& Bontemps, S. 2001, A\&A, 372, L41

Muench, A. A., Alves, J., Lada, C. J., \& Lada, E. A. 2001, ApJ, 558, L51

Muench, A. A., Lada, E. A., Lada, C. J., \& Alves, J. 2002, ApJ, 573, 366

Onishi, T., Kawamura, A., Abe, R., et al. 1999, PASJ, 51, 871

Onishi, T., Mizuno, A., Kawamura, A., Tachihara, K., \& Fukui, Y. 2002, ApJ, 575,950

Padoan, P., \& Nordlund, Å. 2002, ApJ, 576, 870

Salpeter, E. E. 1955, ApJ, 121, 161

Sandell, G., \& Knee, L. B. G. 2001, ApJ, 546, L49

Shu, F. H., Adams, F. C., \& Lizano, S. 1987, ARA\&A, 25, 23

Shu, F. H., Li, Z.-Y., \& Allen, A. 2004, in Star Formation in the Interstellar Medium: in Honor of David Hollenbach, ASP Conf. Ser., 323, 37

Shu, F. H., Ruden, S. P., Lada, C. J., \& Lizano, S. 1991, ApJ, 370, L31

Silverman, B. W. 1986, Density Estimation (London: Chapman and Hall)

Stanke, T., Smith, M. D., Gredel, R., \& Khanzadyan, T. 2006, A\&A, 447, 609

Tachihara, K., Onishi, T., Mizuno, A., \& Fukui, Y. 2002, A\&A, 385, 909

Teixeira, P. S., Lada, C. J., Young, E. T., et al. 2006, ApJ, 636, L45

Testi, L., \& Sargent, A. I. 1998, ApJ, 508, L91 